\title{
Dark-adapted red flash ERGs in healthy adults
}

\author{
R. Hamilton $(\mathbb{D} \cdot$ K. Graham
}

Received: 22 February 2018/ Accepted: 22 May 2018/Published online: 1 June 2018

(C) The Author(s) 2018

\begin{abstract}
Purpose The $\mathrm{x}$-wave of the dark-adapted (DA) ERG to a red flash reflects DA cone function. This exploratory study of healthy adults aimed to investigate changes in the DA red ERG with flash strength and during dark adaptation to optimise visualisation and therefore quantification of the x-wave.

Methods The effect of altering red flash strength was investigated in four subjects by recording ERGs after $20 \mathrm{~min}$ dark adaptation to red flashes $\left(0.2-2.0 \mathrm{~cd} \mathrm{~s} \mathrm{~m}^{-2}\right)$ using skin electrodes and natural pupils. The effect of dark adaptation duration was investigated in 16 subjects during $20 \mathrm{~min}$ in the dark, by recording DA 1.5 red ERGs at 1, 2, 3, 4, 5, 10, 15 and $20 \mathrm{~min}$.

Results For a dark adaption period of $20 \mathrm{~min}$, the $\mathrm{x}$-wave was more clearly visualised to weaker $\left(<0.6 \mathrm{~cd} \mathrm{~s} \mathrm{~m}^{-2}\right)$ red flash strengths: to stronger flashes it became obscured by the b-wave. For red flashes of $1.5 \mathrm{~cd} \mathrm{~s} \mathrm{~m}^{-2}$, the $\mathrm{x}$-wave was most prominent in ERGs recorded after 1-5 min of dark adaptation: with longer dark adaptation, it was subsumed into the b-wave's rising edge.
\end{abstract}

R. Hamilton $(\bowtie) \cdot$ K. Graham

Department of Clinical Physics and Bio-Engineering,

NHS Greater Glasgow and Clyde, Glasgow, UK

e-mail: ruth.hamilton@glasgow.ac.uk

R. Hamilton · K. Graham

College of Medical, Veterinary and Life Sciences,

University of Glasgow, Glasgow, UK
Conclusions This small study suggests that $\mathrm{x}$-wave visibility in healthy subjects after 20 min dark adaptation is improved by using flashes weaker than around $0.6 \mathrm{~cd} \mathrm{~s} \mathrm{~m}^{-2}$; for flash strengths of $1.5 \mathrm{~cd} \mathrm{~s} \mathrm{~m}^{-2}$, $\mathrm{x}$-wave visibility is enhanced by recording after only around 5 min of dark adaptation. No evidence was found that interim red flash ERGs affect the darkadapted state of the normal retina.

Keywords ISCEV standard - Dark-adapted red flash ERG $\cdot \mathrm{x}$-wave $\cdot$ Dark-adapted

\section{Introduction}

The dark-adapted (DA) ERG to a red flash has an initial positive peak called the $\mathrm{x}$-wave $[1,2]$ which is seen only in species with cone-rich retinae [3]. X-wave amplitude is largest to wavelengths around $630 \mathrm{~nm}$ [2-4], and increases during dark adaptation, peaking within a few minutes [4-6]. Its peak time, unlike the b-wave, changes little with wavelength [7]. Visibility of the $\mathrm{x}$-wave can be enhanced by using a dim background to suppress rods $[4,8]$.

No $\mathrm{x}$-wave is evident in protanomalous subjects $[4,7,9]$ nor in subjects with achromatopsia [7, 9]. Conversely, the $\mathrm{x}$-wave is preserved but the $\mathrm{b}$-wave is attenuated or absent in RDH5 retinopathy (fundus albipunctatus) $[10,11]$ and in vitamin A deficiency 
[12]. The DA ERG to a red flash is present-albeit with impaired kinetics [13] —in bradyopsia (RGS9/ R9AP mutation), indicating preserved cone function, whereas the light-adapted white flash ERG is absent, a combination reported to be pathognomonic for the condition [14]. The x-wave is, therefore, interpreted as a measure of dark-adapted cone function. Although a DA red ERG is not part of the ISCEV ERG standard [15], it is used 'sometimes' or 'often' in around half of visual electrophysiology clinics [16]. Red flash strengths of $0.05-2.5 \mathrm{~cd} \mathrm{~s} \mathrm{~m}^{-2}[8,10,17-21]$ have been used, but some studies report clinical use of the red flash ERG without giving flash strength or spectral characteristics, instead describing flash strength "such that in a normal subject the amplitude of the rod component to the red flash is equivalent to that of the rod-specific response to a dim white flash (darkadapted $0.01 \mathrm{~cd} \mathrm{~s} \mathrm{~m}^{-2}$ )" [11, 12, 14, 22].

The $\mathrm{x}$-wave can be swamped by the later, larger rod b-wave, appearing as only a shoulder which hampers quantification [20, 21]. Clearer visualisation and hence quantification of the $\mathrm{x}$-wave might be achieved by a suitable combination of red flash strength and dark adaptation duration. This exploratory study aimed to investigate changes in the DA red ERG with flash strength and during dark adaptation.

\section{Methods}

The study was approved by the Ethics Committee of the College of Medical, Veterinary and Life Sciences, University of Glasgow. Subjects gave informed, written consent.

\section{Subjects}

Sixteen adult subjects (20-58 years old) without selfreported neurological or ocular conditions were recruited without incentive. Inclusion criteria were refractive errors of $<3$ dioptres, and a normal Ishihara colour test result. The sample size was selected to power a concomitant study of shorter dark adaptation for ISCEV standard DA ERGs [23].

\section{Study design}

Subjects were restricted to interior lighting for at least $1 \mathrm{~h}$ prior to any recordings. The test room was artificially lit. The first investigation explored the effect of altering red flash strength on four subjects (all female, aged 22-53). After 20 min dark adaptation, ERGs were recorded as described below from one random eye to red flashes of 0.2 or $0.3-2.0$ phot $\mathrm{cd} \mathrm{s} \mathrm{m}^{-2}$ in 0.1 steps. A white 0.01 phot $\mathrm{cd} \mathrm{s} \mathrm{m}^{-2}$ ERG (DA 0.01 ERG) was also recorded at each step for comparison and to check for any evidence, e.g. reducing amplitude, that the retina was becoming light adapted.

The second investigation explored the effect of altering duration of dark adaptation on the red flash ERGs, and was conducted as part of the concomitant study [23]. In the baseline phase of the experiment (Fig. 1), subjects were dark adapted for $20 \mathrm{~min}$, at the end of which a DA $1.5 \mathrm{~cd} \mathrm{~s} \mathrm{~m}^{-2}$ red flash ERG (followed by ISCEV standard DA 0.01 and DA 3.0 ERGs) was recorded. Subjects were then light adapted to the artificial room lighting for $10 \mathrm{~min}$ before beginning the experimental phase of the protocol. In the experimental phase, dark adaptation was recommenced and interim DA 1.5 red ERGs were recorded during this second 20-min dark adaptation period at 1 , 2, 3, 4, 5, 10, 15 and $20 \mathrm{~min}$. The baseline phase was included in order to compare ERGs recorded after 20 min uninterrupted dark adaptation with those recorded after 20 min dark adaptation punctuated with multiple, interim ERGs, in order to test whether the experimental design itself affected the ERGs.

\section{ERGs}

ERGs were recorded from both eyes using adhesive, disposable skin electrodes placed on the lower lid, referenced to skin electrodes at the ipsilateral temporal orbital rim. A ground electrode was placed on a mastoid. Skin was prepared to ensure low $(<5 \mathrm{k} \Omega)$ and matched impedances; amplifier bandpass was $0.3-300 \mathrm{~Hz}$ (IIR digital, 2 pole Bessel emulations), with a sampling frequency of $1000 \mathrm{~Hz}$. In the interests of investigating protocols with greater patient test acceptability, no dilating drops were used, contrary to the stipulation of the ISCEV standard [15]. Pupil sizes were measured towards the end of the baseline period of dark adaptation using a half-moon rule with $0.5 \mathrm{~mm}$ precision and an infrared camera. Diameters ranged from 7 to $10 \mathrm{~mm}$ (median $8.5 \mathrm{~mm}$ ), very similar to those from an earlier study on similar subjects 


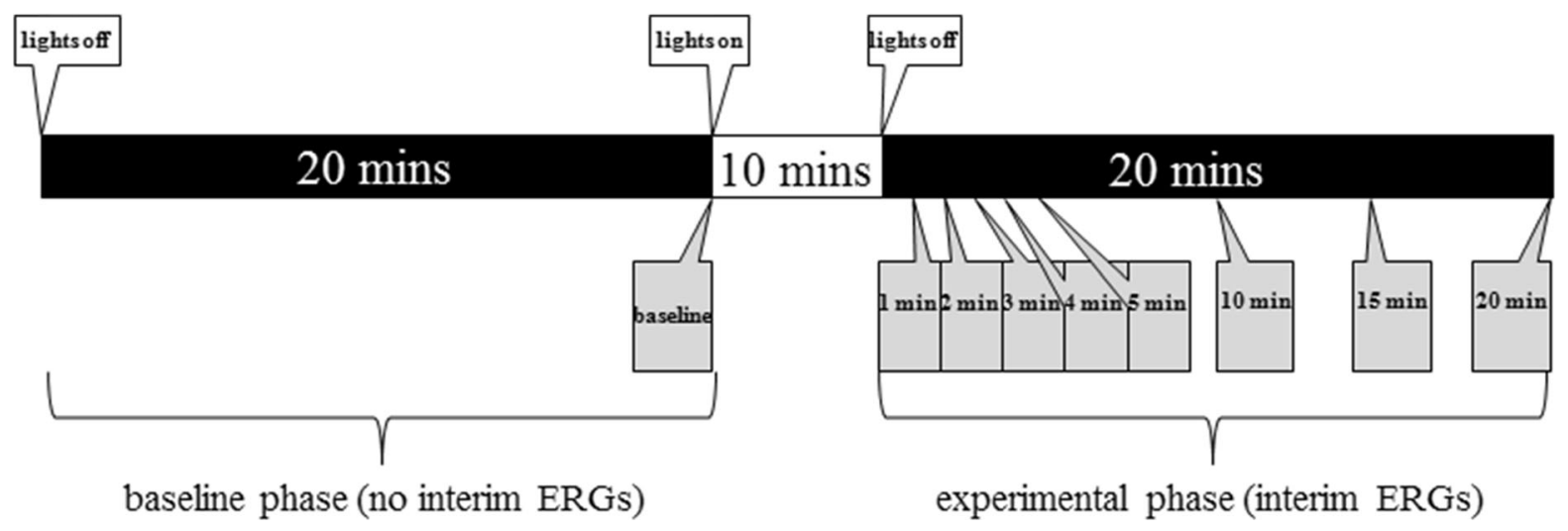

Fig. 1 Timeline illustration of recording protocol. Black and white bands indicate dark and light adaptation, respectively. Boxes below the timeline indicate ERG recordings, labelled by duration of dark adaptation after which they were made

(7-9 mm) with mydriasis [24]. A dim red fixation mark aided eye stability during recordings.

Stimulation and acquisition were driven by a visual electrophysiology system (Espion, Diagnosys LLC, Lowell, MA, USA). Ten ERGs were averaged with an inter-stimulus interval of $1 \mathrm{~s}$ to ensure adequately high SNR since skin electrodes were used. Flashes were generated by LEDs within a ganzfeld (ColorDome, Diagnosys LLC, Lowell, MA, USA) with stated peak wavelength $\lambda=635 \mathrm{~nm}$ and CIE coordinates $x=0.702, y=0.298$. Annual manufacturer's calibration before and after the investigation showed no changes; values were confirmed for white flashes using a photometer (ILT1700, International Light Technologies, MA, USA) in integrating mode. Values given here are manufacturer's nominal values. For the second investigation, a red flash strength of 1.5 (photopic) cd s m${ }^{-2}$ was chosen somewhat arbitrarily, as it fell within the range of flash strengths described elsewhere for use with a preceding 20 min period of dark adaptation (0.05-2.5 $\left.\mathrm{cd} \mathrm{s} \mathrm{m}^{-2}[8,10,17-21]\right)$; additionally, it generated a b-wave of a similar amplitude to that produced by the DA 0.01 ERG white flashes (Fig. 2).

Amplitudes of a-waves were measured from baseline, and $\mathrm{x}$ - and $\mathrm{b}$-waves were measured from a-wave troughs. No systematic inter-ocular ERG differences existed, so parameters from eyes of each subject were averaged [25]. Data were treated nonparametrically because of the small sample size and some skew.

\section{Results}

ERG changes with red flash strength

The red flash ERG grew in amplitude as flash strength increased for all four subjects tested (Fig. 2). The a-wave was present, typically at around 17-20 ms. Two further troughs were evident, typically shallower, at around 25-28 ms and at around 30-37 ms, depending on flash strength. The x-wave was also present, typically at around $45-50 \mathrm{~ms}$, and was more clearly seen at lower than at higher flash strengths: as flash strength increased, it became larger, but was increasingly obscured by the b-wave, appearing as a shoulder on the b-wave rising edge. At lower flash strengths, the b-wave was also clearly visualised at around $100 \mathrm{~ms}$, with a similar form to the b-wave of the dim white flash ERG. As red flash strength increased, the b-wave shortened and merged with the $\mathrm{x}$-wave peak.

The white flash DA 0.01 ERGs recorded at each step showed no evidence of reducing in amplitude over the 10 ERGs used for the average in each step, nor over the whole investigation for any subject, despite a 1 -s inter-stimulus interval, rather than the $2 \mathrm{~s}$ stipulated in the ERG standard [15]. Similarly, the red flash ERGs showed no evidence of reducing in amplitude over the 10 ERGs used for the average in each step, suggesting that, even for relatively strong red flashes, a 1-s inter-stimulus interval was adequate to maintain the dark-adapted state of the retina. 


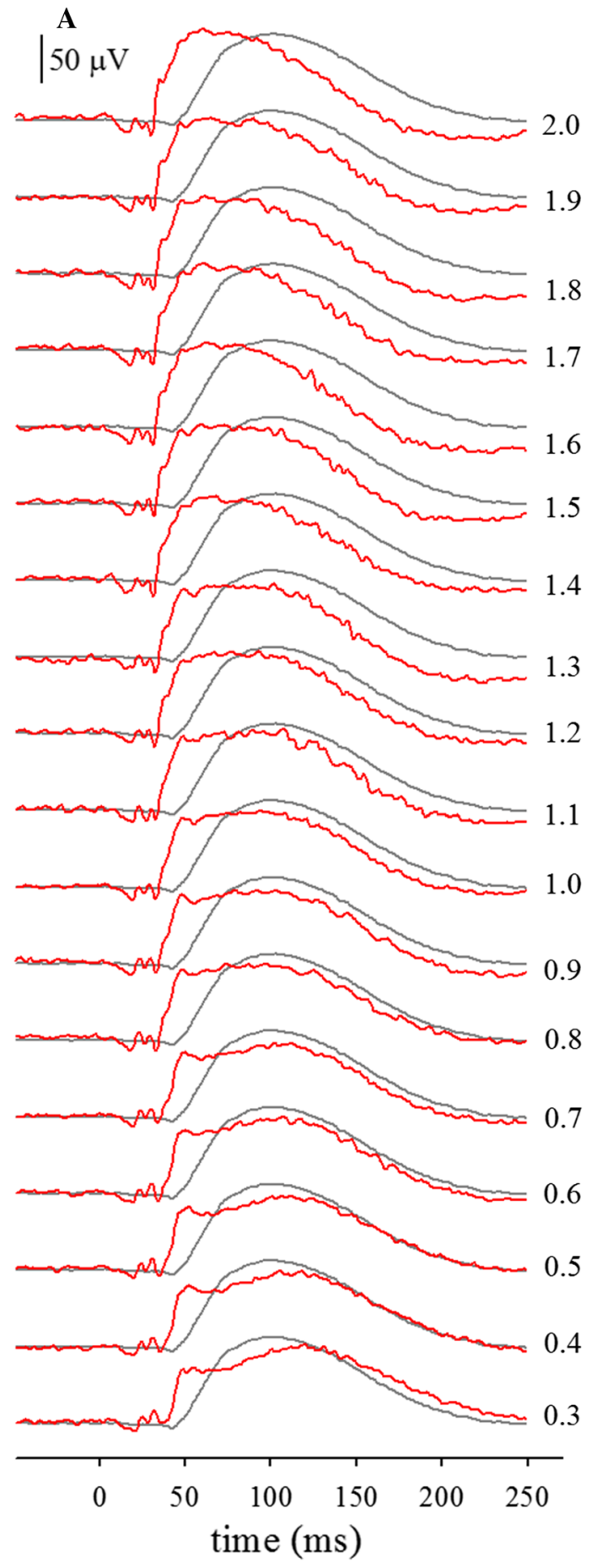

Fig. 2 Illustrative red flash ERGs (red traces) and ISCEV standard DA 0.01 ERGs (grey traces) from subject \#16, recorded after 20 min dark adaptation (a). The numbers to the right of each pair of traces indicate the strength of the red flash in photopic $\mathrm{cd} \mathrm{s} \mathrm{m}^{-2}$. Right panels show effect of red flash
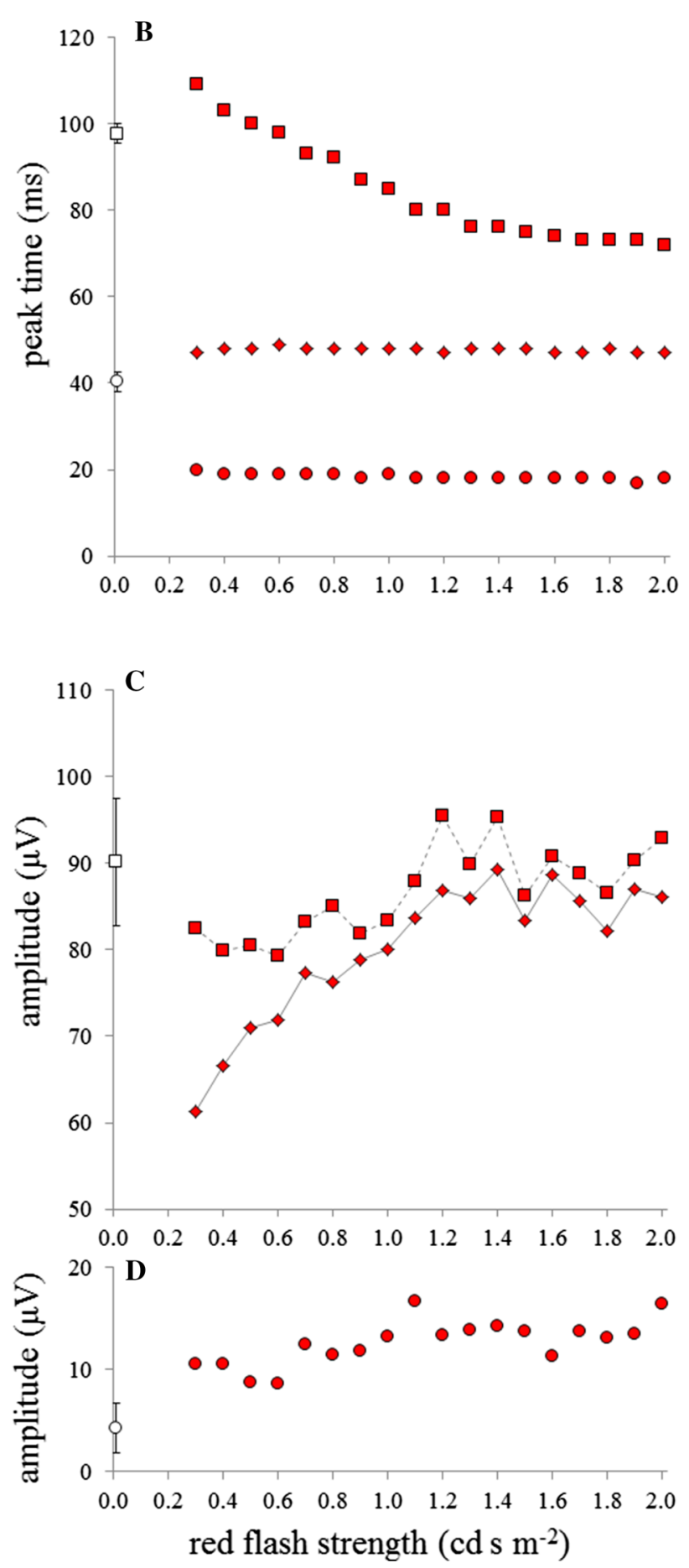

strength on subject \#16's peak times (b); $\mathrm{x}$ and b-wave amplitudes (c) and a-wave amplitudes (d). Circles: a-waves, diamonds: $x$-waves, squares: $b$-waves. Open symbols close to $\mathrm{y}$-axes represent mean (sd) values for DA 0.01 ERGs for comparison 
ERG changes with duration of dark adaptation

Comparing ERGs recorded at the end of the baseline phase with those recorded at the end of the experimental phase revealed no statistically significant differences in ERG parameters (Mann-Whitney $U$ tests, $p$ values all $\geq 0.50$ ), establishing that interim ERGs recorded during the experimental phase did not affect the measured parameters of ERGs recorded at the end of the dark adaptation period (Fig. 3). This also implies that delivering red flashes with 1-s interstimulus intervals does not affect the adaptation state of the retina, notwithstanding the additional white flashes delivered during the experimental phase [23].

The morphology of the DA 1.5 red ERG changes during dark adaptation (Fig. 4). A series of oscillations at the a-wave and rising edge of the b-wave are evident, with the largest positive peak-the X-wavebeing most prominent at around $40 \mathrm{~ms}$ in ERGs recorded after $1-5 \mathrm{~min}$ of dark adaptation. As the presumably rod-driven, later b-wave gains amplitude during dark adaptation, this 40-ms $\mathrm{x}$-wave peak becomes subsumed into the b-wave's rising edge, often being no longer apparent, or present as only a shoulder with no following trough. A later oscillation, usually $>50 \mathrm{~ms}$, is more clearly visualised as the $\mathrm{x}$-wave than the 40-ms peak in ERGs recorded after 10-20 min of dark adaptation.

The development of the DA red 1.5 ERG during dark adaptation was quantified by normalising individual subject's ERG parameters to those of their

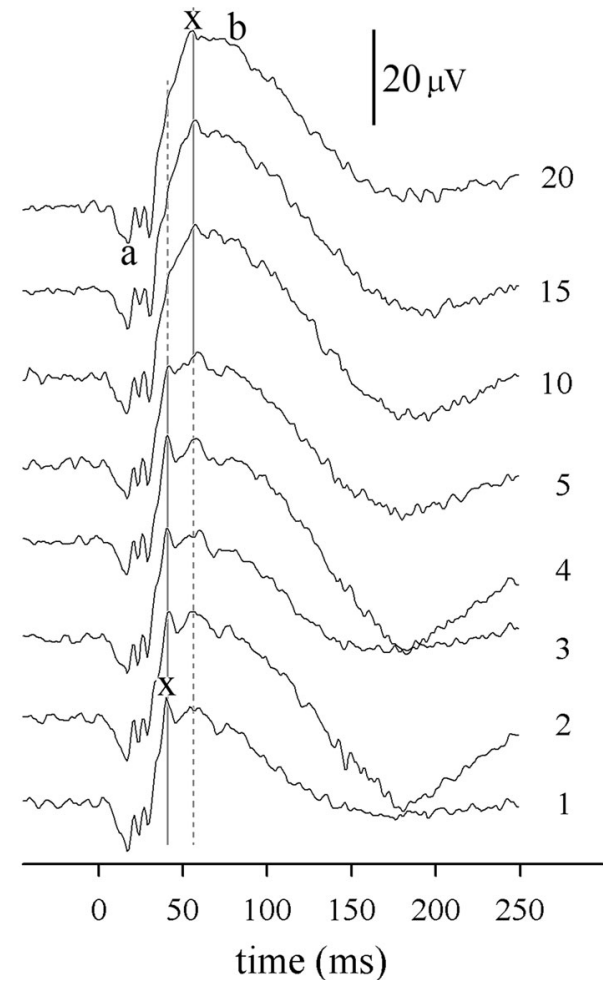

Fig. 4 Illustrative DA 1.5 red ERGs from a typical subject (\#8). The numbers to the right of each trace indicate the duration of dark adaptation (minutes) before each ERG. Vertical grey lines mark the two positive peaks which exchange dominance (largest amplitude) as dark adaptation proceeds. The largest peak is measured as the $\mathrm{x}$-wave (continuous line). Note the triple trough which forms the a-wave, with troughs typically at around 17, 24 and $31 \mathrm{~ms}$
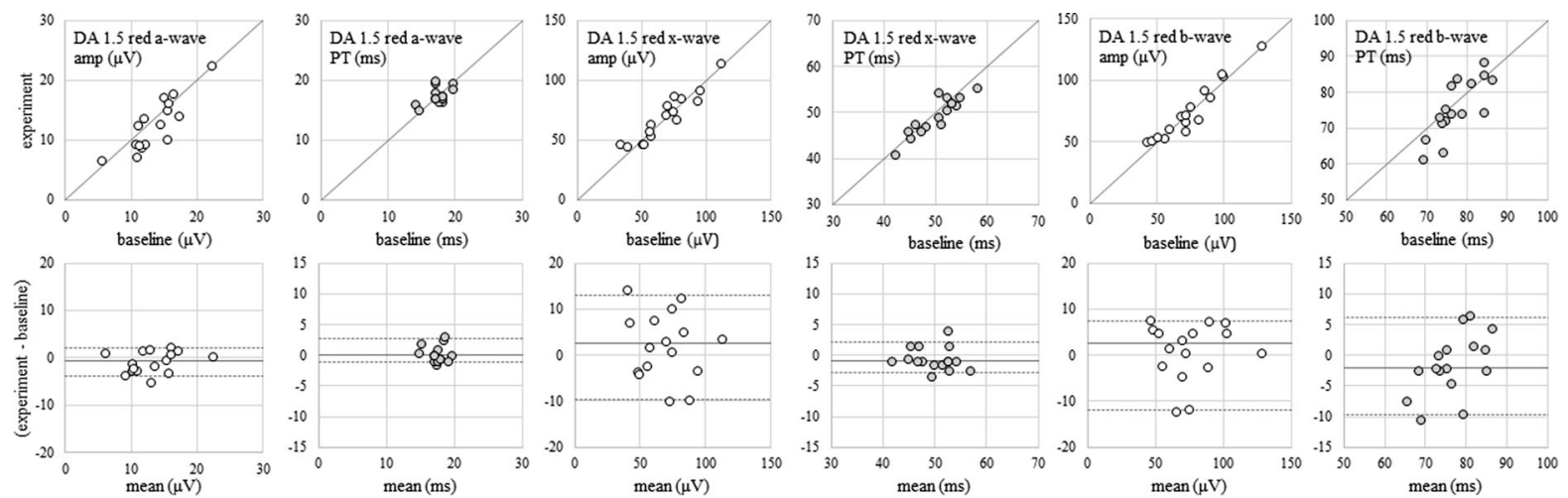

Fig. 3 Upper panels: scatterplots of ERG parameters recorded at the end of baseline phase versus end of experimental phase for all 16 subjects. Grey diagonal lines indicate equality. Lower panels: corresponding difference plots. Grey horizontal lines

indicate the median difference between experimental and baseline recordings, and grey dashed lines indicate the 5 th and 95th percentiles of the difference 
Fig. 5 DA red 1.5 ERG changes during dark adaptation. Left: amplitudes. Right: peak times. Circles: individual subject's normalised data points; triangles: median values; dashed lines: 95\% prediction intervals of growth curves fitted to median data; solid horizontal line highlights the $100 \%$ level. Data are normalised relative to values after 20 min dark adaptation, hence the lack of variability at $20 \mathrm{~min}$. Note change of scale for a-wave amplitudes
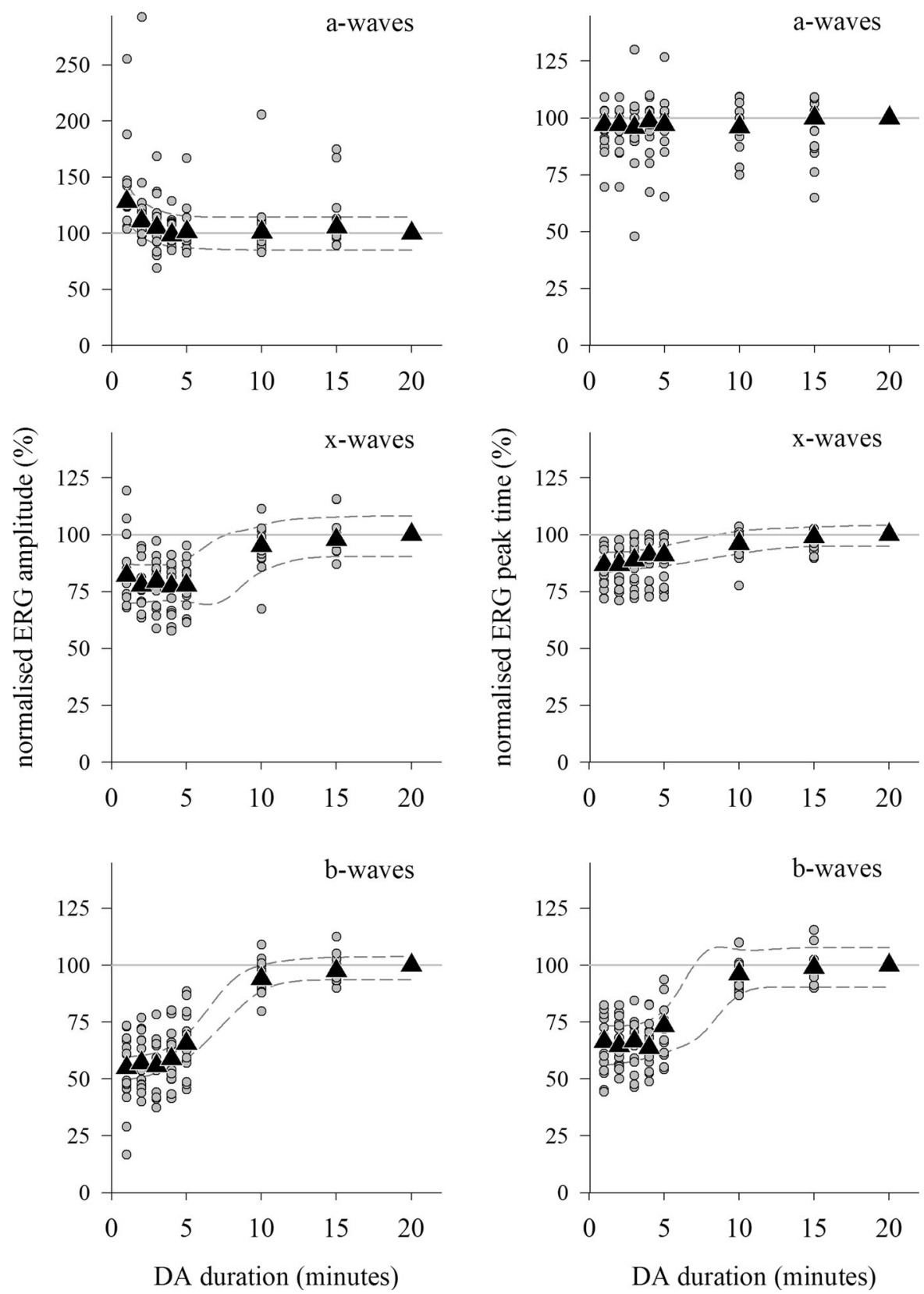

20 min DA ERG (Fig. 5). The red a-wave amplitude reduced a little over the first 3 min of dark adaptation, while peak times did not change at all over the whole $20 \mathrm{~min}$. The $\mathrm{x}$-wave amplitude increased, and peak time lengthened between five and 10 min of dark adaptation, primarily due to the later peak at $\sim 50 \mathrm{~ms}$ becoming dominant as the growing $b$-wave obscured the earlier $\mathrm{x}$-wave peak at $\sim 40 \mathrm{~ms}$. The b-wave amplitude increased, and peak time shortened with lengthening dark adaptation as might be expected for a rod system b-wave.

Summarised reference data are presented in Table 1 for the DA 1.5 red flash ERG after 5, 10 and 20 min of dark adaptation: data are presented as ranges as the sample size is inadequate for percentiles with confidence intervals [26]. These data do not change substantially with duration of dark adaptation, except for the $\mathrm{x}$-wave peak time which more tightly 
Table 1 Summarised reference data (ranges) for DA 1.5 red flash ERGs, skin electrodes, undilated pupils, $N=16$ subjects

\begin{tabular}{|c|c|c|c|c|c|c|}
\hline \multirow[t]{2}{*}{ Duration of dark adaptation (min) } & \multicolumn{2}{|l|}{ a-wave } & \multicolumn{2}{|l|}{$\mathrm{x}$-wave } & \multicolumn{2}{|l|}{ b-wave } \\
\hline & Amplitude $(\mu \mathrm{V})$ & PT (ms) & Amplitude $(\mu \mathrm{V})$ & PT (ms) & Amplitude $(\mu \mathrm{V})$ & PT $(\mathrm{ms})$ \\
\hline 5 & $5.2-24$ & $15-20$ & $31-100$ & $41-50$ & $21-97$ & $62-89$ \\
\hline 10 & $6.3-25$ & $15-19$ & $38-110$ & $41-58$ & $40-128$ & $66-92$ \\
\hline 20 & $6.6-22$ & $15-20$ & $45-114$ & $41-56$ & $50-129$ & $62-89$ \\
\hline
\end{tabular}

$P T$ peak time

defined after $5 \mathrm{~min}$ in the dark than after longer dark adaptation.

\section{Discussion}

In this small, exploratory study of healthy adult subjects, we found that after 20 min of dark adaptation, the $\mathrm{x}$-wave was more clearly visualised with weaker (about $0.6 \mathrm{~cd} \mathrm{~s} \mathrm{~m}^{-2}$ or less) than with stronger flashes, as described elsewhere [18]. We also found that that with a relatively strong $1.5 \mathrm{~cd} \mathrm{~s} \mathrm{~m}^{-2}$ red flash, the $\mathrm{x}$-wave was more clearly visualised after shorter (about $5 \mathrm{~min}$ or less) than longer dark adaptation, also confirming findings elsewhere [6].

The subjects in this study were mostly in their second or third decades, so findings cannot be generalised to all age groups. As an exploratory study, we chose a minimally invasive protocol with noncorneal electrodes and no mydriasis: this will have resulted in higher signal-to-noise ratios than usually found with corneal electrodes. This is unlikely to affect conclusions since mostly relative outcome measures were used, but signal noise increases uncertainty of peak labelling in some instances.

The DA red flash ERG is quite widely used, and the International Society for Clinical Electrophysiology of Vision has recently prepared a new Extended Protocol to inform current and potential users. Its utility lies in an extant $\mathrm{x}$-wave revealing the presence of functioning, dark-adapted cones. Thus, the visibility of the $x$-wave is of primary importance for the optimisation of protocols. The current findings suggest that $\mathrm{X}$-wave visibility in normal subjects is impaired by flashes stronger than around $0.6 \mathrm{~cd} \mathrm{~s} \mathrm{~m}^{-2}$ (for $20 \mathrm{~min}$ dark adaptation), or by dark adaptation longer than around $5 \mathrm{~min}$ (for flash strengths of $1.5 \mathrm{~cd} \mathrm{~s} \mathrm{~m}^{-2}$ ). Other combinations remain uninvestigated. It seems feasible that the ISCEV standard dark-adapted ERG protocol could incorporate a dim red flash ERG delivered at some point during the currently stipulated 20 min of dark adaptation, adding no further burden of time to the patient or tester. These data also suggest that additional flashes, even delivered once per second, are unlikely to affect the dark-adapted state of the normal retina, although this may not be the case for patients with retinal dysfunction.

\section{Compliance with ethical standards}

Conflict of interest The authors declare that they have no conflicts of interest.

Ethical approval All procedures performed in studies involving human participants were in accordance with the ethical standards of the institutional and/or national research committee and with the 1964 Helsinki declaration and its later amendments or comparable ethical standards.

Statement of human rights All procedures performed in studies involving human participants were in accordance with the ethical standards of the institutional and/or national research committee and with the 1964 Helsinki Declaration and its later amendments or comparable ethical standards.

Statement on the welfare of animals This article does not contain any studies with animals performed by any of the authors.

Informed consent Informed consent was obtained from all individual participants included in the study.

Open Access This article is distributed under the terms of the Creative Commons Attribution 4.0 International License (http:// creativecommons.org/licenses/by/4.0/), which permits unrestricted use, distribution, and reproduction in any medium, provided you give appropriate credit to the original author(s) and the source, provide a link to the Creative Commons license, and indicate if changes were made. 


\section{References}

1. Motokawa K, Mita T (1942) Über eine einfachere Untersuchungsmethode und Eigenschaften der Aktionsströme der Netzhaut des Menschen. Tohoku J Exp Med 42:114-133. https://doi.org/10.1620/tjem.42.114

2. Adrian ED (1945) The electric response of the human eye. J Physiol 104:84-104. https://doi.org/10.1113/jphysiol. 1945.sp004109

3. Adrian ED (1946) The rod and cone components in the electrical response of the human eye. J Physiol 104:84-104. https://doi.org/10.1113/jphysiol.1945.sp004109

4. Armington JC, Johnson EP, Riggs LA (1952) The scotopic a-wave in the electrical response of the human retina. J Physiol 118:289-298

5. Auerbach E, Burian HM (1955) Studies on the photopicscotopic relationships in the human electroretinogram. Am J Ophthalmol 40(5):42-60

6. Kawabata H (1963) Changes in the human electroretinogram during early dark adaptation. J Opt Soc Am 53:386-390. https://doi.org/10.1364/josa.53.000386

7. Von Schubert G, Bornschein H (1952) Beitrag zur Analyse des menschlichen Elektroretinogramms. Ophthalmologica 123(6):396-412

8. Miyake Y (2006) Electrodiagnosis of retinal diseases. Springer, Yokyo, pp 16-17. ISBN 4-431-25466-8

9. François J, Verriest G, de Rouck A (1956) Pathology of the $\mathrm{X}$-wave of the human electroretinogram 1. Red-blindness and other congenital functional abnormalities. Br J Ophthalmol 40:439-443

10. Liu X, Liu L, Li H, Jiang R, Sui R (2015) RDH5 retinopathy (fundus albipunctatus) with preserved rod function. Retina 35:582-589. https://doi.org/10.1097/iae.0000000000000319

11. Sergouniotis PI, Sohn EH, Li Z, McBain VA, Wright GA, Moore AT, Robson AG, Holder GE, Webster AR (2011) Phenotypic variability in RDH5 retinopathy (fundus albipunctatus). Ophthalmology 118:1661-1670. https://doi. org/10.1016/j.ophtha.2010.12.031

12. McBain VA, Egan CA, Pieris SJ, Supramaniam G, Webster AR, Bird AC, Holder GE (2007) Functional observations in vitamin A deficiency: diagnosis and time course of recovery. Eye 21:367-376. https://doi.org/10.1038/sj.eye.6702212

13. Nishiguchi KM, Sandberg MA, Kooijman AC, Martemyanov KA, Pott JWR, Hagstrom SA, Arshavsky VY, Berson EL, Dryja TP (2004) Defects in RGS9 or its anchor protein R9AP in patients with slow photoreceptor deactivation. Nature 427:75-78. https://doi.org/10.1038/ nature 02170
14. Vincent A, Robson AG, Holder GE (2013) Pathognomonic (diagnostic) ERGs: a review and update. Retina 33:5-12. https://doi.org/10.1097/iae.0b013e31827e2306

15. McCulloch DL, Marmor MF, Brigell MG, Hamilton R, Holder GE, Tzekov R, Bach M (2015) ISCEV Standard for full-field clinical electroretinography (2015 update). Doc Ophthalmol 130:1-12. https://doi.org/10.1007/s10633-0149473-7

16. McCulloch DL (2012) ISCEV ERG Survey 2012, International Society for Clinical Electrophysiology of Vision. www.iscev.org/varia/2013/ISCEV_ERG_Survey2012.pdf. Accessed 21 Feb 2018

17. Mizunoya S, Kuniyoshi K, Arai M, Tahara K, Hirose T (2001) Electroretinogram contact lens electrode with tricolor light-emitting diode. Acta Ophthalmol Scand 79(5):497-500

18. Chen LY, Png R, Mathur R, Chia A (2015) Scotopic red ERG findings. ISCEV Symposium, Ljubljana, Slovenia, June 2015. Doc Ophthalmol 130(1S):31. https://doi.org/10. 1007/s10633-015-9500-3

19. Lovasik JV, Kothe AC, Kergoat H (1992) Improving the diagnostic power of electroretinography by transient alteration of the ocular perfusion pressure. Optom Vis Sci 69(2):85-94

20. Lim S-H, Ohn Y-H (2005) Study of blue and red flash in dark-adapted electroretinogram. Korean J Ophthalmol 19:106-111. https://doi.org/10.3341/kjo.2005.19.2.106

21. Chia A, Png R, Mathur R (2014) Scotopic red response: rod and cone components. ISCEV symposium, Boston, USA. Doc Ophthalmol 129(1S):42. https://doi.org/10.1007/ s10633-014-9441-2

22. Cheng JYC, Luu CD, Yong VHK, Mathur R, Aung T, Vithana EN (2007) Bradyopsia in an Asian man. Arch Ophthalmol 125:1138-1140. https://doi.org/10.1001/archopht.125.8.1138

23. Hamilton R, Graham K (2016) Effect of shorter dark adaptation on ISCEV standard DA 0.01 and DA 3 skin ERGs in healthy adults. Doc Ophthalmol 133:11-19. https://doi.org/10.1007/s10633-016-9554-X

24. Abdlseaed A Al (2014) Pupil dilation, light source, gender and pigmentation effects on normal adult human ERGs, and an exploration of short latency VEPs. PhD thesis, Glasgow Caledonian University

25. Armstrong RA (2013) Statistical guidelines for the analysis of data obtained from one or both eyes. Ophthalmic Physiol Opt 33:7-14. https://doi.org/10.1111/opo.12009

26. Horowitz GL (2010) EP28-A3C defining, establishing, and verifying reference intervals in the clinical laboratory; approved guideline, 3rd edn. Clinical and Laboratory Standards Institute, San Diego 